\title{
Análise do monitoramento ambiental da incineração de resíduos sólidos urbanos na Europa ea necessidade de alterações na legislação brasileira
}

\author{
Environmental monitoring analysis of municipal solid waste incineration in \\ Europe and the needs for changes in brazilian legislation
}

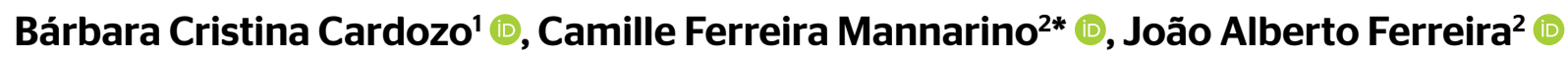

\begin{abstract}
RESUMO
A incineração é uma tecnologia de tratamento térmico de resíduos sólidos cujo principal objetivo é a redução do volume e da massa dos resíduos a serem depositados em aterros. Os incineradores construídos nas últimas décadas trazem a possibilidade de recuperação energética dos resíduos, na forma de vapor e/ou energia elétrica, entretanto essa tecnologia ainda gera questionamentos sobre possíveis impactos ambientais relacionados às suas emissões. A incineração éregulada na Europa por legislação específica que, entre outras questões, obriga a divulgação de dados de monitoramento ambiental de incineradores. No presente trabalho, são analisados dados de emissões atmosféricas de incineradores associados à Confederation of European Wasteto-Energy Plants (CEWEP), por meio de buscas nos websites dos respectivos incineradores, em artigos científicos e relatórios técnicos. Foram examinados dados de monitoramento de todos os poluentes de medição contínua e periódica definidos na legislação da UE, em séries temporais de 2010 a 2017. Todos os valores médios anuais de emissões nos incineradores analisados estiveram abaixo dos limites definidos pela diretiva europeia, com pequena variação ao longo dos anos observados. A comparação dos padrões de emissão europeus com os brasileiros mostra a necessidade de mudança na legislação local referente à incineração para que, caso essa tecnologia seja implementada no Brasil, ela possa operar respeitando limites seguros, com garantia de minimização de impactos ao ambiente e às populações do entorno. Palavras-chave: tratamento de resíduos; incineração de resíduos sólidos urbanos; monitoramento ambiental; emissões atmosféricas.
\end{abstract}

\begin{abstract}
Incineration is a solid waste treatment technology whose main purpose is to reduce the volume and mass of the waste to be disposed of in landfills. The incinerators built in the last decades bring the possibility of energy recovery from waste, in the form of steam and/or electric energy. However, this technology still raises questions about possible environmental impacts related to its emissions. Incineration is regulated in Europe through specific legislation which, among other issues, requires the disclosure of environmental monitoring data for incinerators. In the present work, atmospheric emissions data from incinerators associated with the Confederation of European Waste-to-Energy Plants (CEWEP) are analyzed by searching the websites of the respective incinerators, scientific articles and technical reports. Monitoring data on all continuous and periodic measured pollutants defined in the legislation in time series from 2010 to 2017 were analyzed. All annual average emission values of the incinerators analyzed were below the limits defined by the European Union's Directive, with little variation over the observed years. The comparison of European with Brazilian emission standards shows the need for a change in local legislation on incineration so that, if this technology is implemented in Brazil, it will operate within safe limits, with a guarantee of minimizing impacts on the environment and surrounding populations.
\end{abstract}

Keywords: waste treatment; municipal solid waste incineration; environmental monitoring; atmospheric emissions.

\section{INTRODUÇÃO}

Embora a incineração seja uma tecnologia de tratamento térmico consolidada nos sistemas de gerenciamento integrado de resíduos sólidos urbanos (RSU), nos países desenvolvidos, especialmente nos europeus, ela ainda desperta dúvidas e discussões sobre seus riscos ambientais e ameaças à saúde pública (VAN DIJK; VAN DOORN; VAN ALFEN, 2015). Observa-se uma tendência de evolução dos

'Universidade do Estado do Rio de Janeiro - Rio de Janeiro (RJ), Brasil.

²Fundação Oswaldo Cruz - Rio de Janeiro (RJ), Brasil.

*Autora correspondente: camille.mannarino@gmail.com

Conflitos de interesse: os autores declaram não haver conflito de interesses.

Financiamento: nenhum.

Recebido: 11/O2/2019 - Aceito: 13/11/2O19 - Reg. ABES: 20190040 
sistemas de gestão de resíduos em países e regiões do mundo, conformando a necessidade de preparação para que as novas tecnologias, quando forem consideradas, estejam reguladas de forma a assegurar a qualidade da operação e, especialmente, dos sistemas de controles ambientais (QUICKER; HEE, 2013; SCHNEIDER; RAGOSSNIG, 2013).

Além de resultar em redução de massa e volume de RSU de cerca de 70 e $90 \%$, respectivamente, a incineração é vista por diversos autores como forma segura de recuperação de energia, pela qual também ocorre a minimização da periculosidade dos resíduos (NZIHOU et al., 2012; USHIMA; SANTOS, 2018). À medida que a incineração dos RSU se foi fazendo presente na gestão de RSU nos países europeus, a preocupação com as questões ambientais foi exigindo ações mais restritivas das autoridades com relação às emissões dos incineradores.

A Diretiva 75/2010 da União Europeia (UE) apresenta um capítulo específico que estabelece as condições, os requisitos técnicos e os valores dos limites de emissão de parâmetros para a regulação da incineração. Uma das obrigações previstas pela Diretiva é a divulgação de dados de monitoramento para instalações com capacidade nominal mínima de recepção de resíduos acima de duas toneladas por hora. Esses dados devem ser lançados preferencialmente em relatórios e fornecer informações sobre funcionamento, controle e emissões atmosféricas e de efluentes, sempre em comparação aos valores limites legais (UE, 2010). A obrigação de tornar públicas as informações das plantas de incineração garante à população o poder de fiscalização e acompanhamento das condições de operação da tecnologia.

O presente trabalho tem como objetivo analisar informações e dados publicados em relatórios divulgados por operadores de unidades de incineração na Europa em seus websites, com foco principal nas emissões atmosféricas. Buscou-se fazer uma análise da legislação brasileira sobre o assunto, de forma a contribuir para uma visão mais ampla das alterações necessárias, com vistas à construção de referências legais que balizem a implantação adequada, sob o ponto de vista ambiental, dessa tecnologia no Brasil.

\section{METODOLOGIA}

A base de dados escolhida para a seleção inicial dos incineradores foi o website da Confederação Europeia de Plantas de Recuperação de Energia Proveniente de Resíduos (Confederation of European Waste-to-Energy Plants - CEWEP), o qual foi acessado entre fevereiro e junho de 2018. Buscou-se identificar o número de unidades de incineração de resíduos sólidos urbanos por país. Identificou-se o total de 522 plantas de incineração nos 22 países-membros da CEWEP. A avaliação preliminar dos dados disponibilizados revelou a homogeneidade existente entre eles, em função de se ter estabelecido que a amostra a ser trabalhada seria da ordem de $20 \%$ do total de incineradores.

No mesmo sentido, definiu-se que a amostra com a qual se trabalharia seria de 10 incineradores por país, naqueles em que há um número superior a esse de plantas. Em 10 países vinculados à CEWEP em que o número de plantas existentes era menor que 10, considerou-se a totalidade existente. Buscaram-se plantas distribuídas por diferentes regiões de cada país e que possuíssem as maiores capacidades de recepção de resíduos. Um critério de seleção foi a escolha de plantas que disponibilizassem as informações de interesse em seus websites.
Esse procedimento resultou em uma amostra inicial de 136 unidades de incineração, distribuídas por 22 países: França, Alemanha, Itália, Inglaterra, Suécia, Suíça, Dinamarca, Noruega, Bélgica, Holanda, Espanha, Áustria, Finlândia, Polônia, República Checa, Eslováquia, Portugal, Hungria, Lituânia, Estônia, Irlanda e Principado de Andorra (Anexo 1).

Foram coletados dados secundários referentes às características técnicas dos incineradores, como tipo de forno, capacidade de recepção e tipo de resíduos recebido, disponibilização de dados sobre os subprodutos e efluentes gerados no processo. Todas as informações foram compiladas em tabelas do programa Excel (Microsoft Excel ${ }^{\circledR}$ 2013).

A Diretiva 2010/75 da UE foi utilizada como referência para emissões atmosféricas. Ela divide os poluentes atmosféricos em dois grupos: os de monitoramento contínuo, com medições em tempo real de suas emissões; e os de medição periódica, com o mínimo duas medições anuais de suas emissões.

Para os poluentes de medição contínua, óxidos de nitrogênio $\left(\mathrm{NO}_{\mathrm{x}}\right)$, monóxido de carbono (CO) e material particulado (MP), foram construídas séries temporais de distribuição de valores anuais de emissões para o período de 2010 a 2017, de forma a identificar variações na qualidade das emissões ao longo desses anos. Esses parâmetros foram escolhidos por permitirem a análise tanto de boas práticas de combustão quanto dos equipamentos de tratamento dos gases. Dioxinas e furanos (PCDD/F), apesar de não serem poluentes com a exigência de monitoramento contínuo, também foram analisados para esse período de oito anos, pela sua importância para o meio ambiente e saúde pública.

Em razão de diferenças nos períodos de disponibilização de dados de monitoramento por parte dos incineradores, o número de plantas analisadas em cada ano variou. Definiu-se que, para ser incluída na amostra, a planta deveria ter dados disponíveis para ao menos cinco dos oito anos considerados (2010 a 2017). Nessa etapa, trabalhou-se com 43 incineradores em 14 países.

Os poluentes de medição periódica Antimônio (Sb), Arsênio (As), Chumbo $(\mathrm{Pb})$, Cromo $(\mathrm{Cr})$, Cobalto $(\mathrm{Co})$, Cobre $(\mathrm{Cu})$, Manganês $(\mathrm{Mn})$, Níquel $(\mathrm{Ni})$ Vanádio (V), Cádmio (Cd), Tálio ( $\mathrm{Tl})$ e Mercúrio $(\mathrm{Hg})$ foram analisados para o período de 2015 a 2017. Nessa análise, incluíram-se também os parâmetros de monitoramento contínuo, perfazendo todos os critérios definidos na Diretiva 2010/75/UE.

Mesmo em um período menor de análise (três anos), houve diferença entre o número de dados de monitoramento disponibilizados por parte dos incineradores em cada ano. Foram consideradas, na amostra, todas as plantas que disponibilizassem dados de monitoramento para ao menos um ano do período considerado (2015 a 2017). Nessa etapa, trabalhou-se com 76 incineradores de 17 países.

Os dados de emissão atmosférica foram coletados e agrupados sob a forma de tabelas e gráficos do tipo box plot, utilizando-se o programa GraphPad Prism 7.0 para Mac (GraphPad Software, La Jolla, California, USA, www.graphpad. com na versão de teste gratuito). Gráficos box plot mostram a dispersão dos dados e permitem análise exploratória e comparação entre eles. Nos gráficos apresentados, as médias são indicadas pelo símbolo +, as medianas pela linha no interior da caixa e os outliers pelos asteriscos *

Após a análise dos dados reais de monitoramento, busca-se comparar as legislações europeia e brasileiras pertinentes às emissões atmosféricas de incineradores, fazendo uma análise crítica das fragilidades locais. Por fim, o trabalho traz uma discussão sobre questões relativas à implantação de incineradores no Brasil, sob o ponto de vista ambiental. 


\section{RESULTADOS E DISCUSSÃO}

Analisando-se as características técnicas dos incineradores quanto ao tipo de forno, todos os da amostra utilizam tecnologia de grelha e recebem predominantemente RSU (EUROPEAN COMMISSION, 2006; WISSING; WIRTZ; SCHERER, 2017). No entanto, podem receber outros tipos de resíduos com características semelhantes, em menor quantidade. Dos 136 incineradores analisados, $62 \%$ informam que recebem somente RSU e $38 \%$ recebem também outros tipos, como os resíduos industriais não perigosos e os comerciais. Em relação à capacidade de recepção, 37\% dos incineradores analisados recebem entre 301 e 700 toneladas por dia, 30\% recebem acima de mil toneladas dia e apenas $15 \%$ recebem até 300 toneladas por dia de resíduos.

A divulgação dos dados de monitoramento de emissões atmosféricas ocorre principalmente em relatórios anuais em $44,19 \%$ dos incineradores analisados. Outros $51,16 \%$ divulgam os dados mensalmente ou diariamente e $4,65 \%$ o fazem trimestral ou semestralmente em seus websites.

Inicialmente, foram avaliados os dados divulgados referentes aos parâmetros considerados de monitoramento contínuo.

Os óxidos de nitrogênio $\left(\mathrm{NO}_{\mathrm{x}}\right)$ referem-se aos compostos óxido nítrico (NO) e dióxido de nitrogênio $\left(\mathrm{NO}_{2}\right)$ e estão presentes nos materiais plásticos, resíduos têxteis e nas proteínas dos resíduos orgânicos que compõem os RSU (SVOBODA; BAXTER; MARTINEC, 2006).

A distribuição dos valores de emissão de $\mathrm{NO}_{x}$ dos incineradores analisados pode ser observada na Figura 1. As médias anuais variaram entre $84.45 \mathrm{mg} . \mathrm{Nm}^{-3}$, em 2017, e $110.01 \mathrm{mg} \cdot \mathrm{Nm}^{-3}$, em 2011. Esses valores estão abaixo dos encontrados por Waldner et al. (2013) e por Nixon et al. (2013): $140 \mathrm{mg} \cdot \mathrm{Nm}^{-3} \mathrm{em}$ um incinerador de grelha, na Itália, e 180 e 146 mg.m ${ }^{-3}$ em dois incineradores, também de grelha, na Inglaterra.

De acordo com Nixon et al. (2013), $\mathrm{NO}_{\mathrm{x}}$ é o parâmetro medido continuamente que mais se aproxima dos limites de emissão estabelecidos pela Diretiva europeia. Os resultados apresentados na Figura 1 mostram distribuição constante ao longo dos anos, sem a ocorrência de valores acima do limite legal de $200 \mathrm{mg} \cdot \mathrm{Nm}^{-3}$. Os valores médios anuais de emissão de $\mathrm{NO}_{\mathrm{x}}$ são aproximadamente $40 \%$ inferiores ao limite legal de emissão para esse parâmetro.

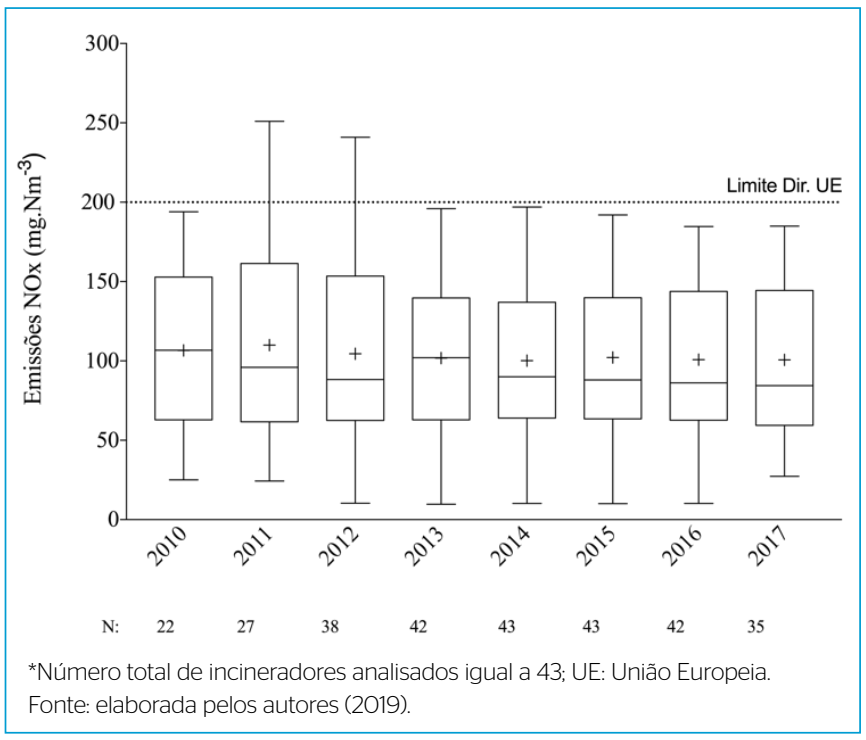

Figura 1 - Distribuição dos valores de emissão de óxidos de nitrogênio*.
O monóxido de carbono ( $\mathrm{CO}$ ) é formado principalmente por materiais que não sofreram combustão completa, por causa da redução da quantidade de oxigênio no processo de queima (STROBEL; WALDNER; GABLINGER, 2018). A melhor forma de reduzir a geração desse poluente é adotando boas práticas de combustão, principalmente, mantendo níveis adequados de oxigênio no processo.

A Figura 2 apresenta a distribuição dos valores de emissão do CO dos incineradores analisados, no período de 2010 a 2017. Pode ser observada a distribuição constante das emissões, com maior densidade de dados abaixo de 20 mg. $\mathrm{Nm}^{-3}$. Pontualmente, nos anos de 2011, 2013 e 2016, alguns incineradores tiveram emissões acima do limite permitido. Os valores médios de CO, em todos os anos avaliados, representam menos de $20 \%$ do valor de referência de emissão, $50 \mathrm{mg} . \mathrm{Nm}^{-3}$.

MP pode ser formado por fragmentação ou por nucleação (CARBONE et al., 2008), durante o processo de combustão dos resíduos. Sua concentração e tamanho podem variar de acordo com o material do resíduo e com o sistema de tratamento de gases do incinerador (KUMAR et al., 2013). As cinzas de instalações modernas de incineração de resíduos são materiais finamente particulados, com tamanhos de partícula na ordem de $50 \mu \mathrm{m}$ ou inferior.

Todos os valores médios anuais, apresentados na Figura 3, assemelham-se ao encontrado por Buonanno et al. (2009) em um incinerador na Itália, de 0,2 mg. $\mathrm{Nm}^{-3}$, e encontram-se em torno de sete vezes abaixo do limite de emissão estabelecido pela Diretiva da UE (10 mg.Nm ${ }^{-3}$ ). Os valores de emissão de MP pelos incineradores podem ser considerados uma pequena fração do total medido no ambiente e pouco significativos quando comparados com outras fontes de emissão, como veículos automotores e plantas de geração de energia que se utilizam de combustíveis fósseis (CASS et al., 2000).

É de extrema importância que as emissões de material particulado sejam baixas, visto que elas carreiam metais potencialmente tóxicos, hidrocarbonetos policíclicos aromáticos, $\mathrm{PCDD} / \mathrm{F}$, que se condensam na superfície do material particulado durante o processo de combustão (VEHLOW; DALANGER, 2011).

As emissões de MP por incineradores podem ser comparadas às presentes em ambientes rurais, com a concentração do número de partícula de aproximadamente $10^{3} \mathrm{~cm}^{-3}$ (BUONANNO et al., 2011; KUMAR et al., 2013). Nenhum dos incineradores analisados apresentou valores de emissão superiores ao limite

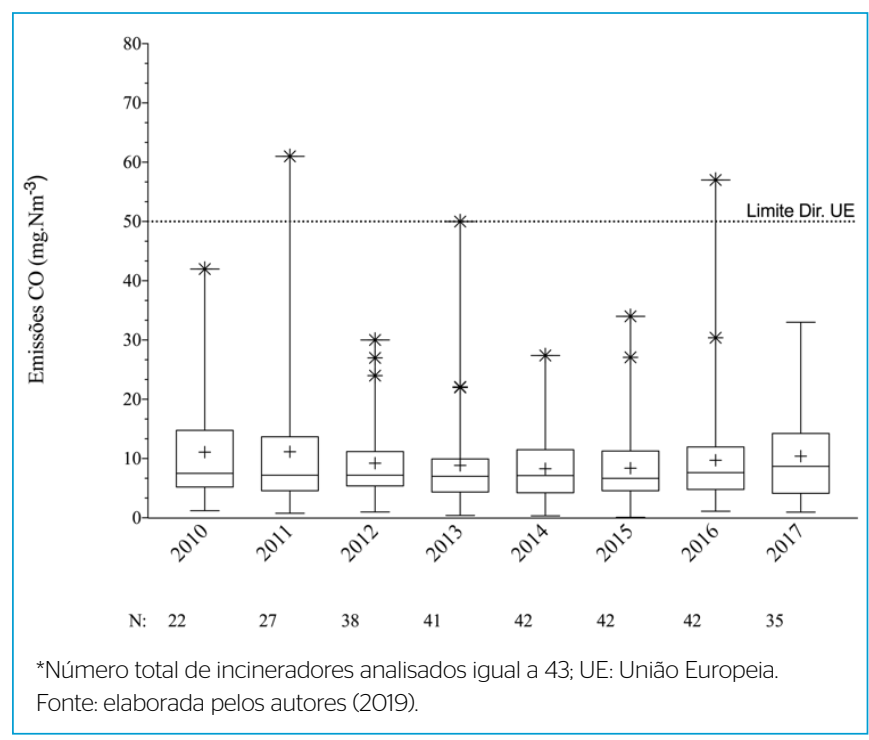

Figura 2 - Distribuição dos valores de emissão do monóxido de carbono*. 
permitido pela Diretiva 2010/75/UE. Os maiores valores foram observados, de forma pontual, nos anos de 2012 e 2015. Os valores médios anuais de MP são aproximadamente $90 \%$ inferiores ao limite legal de emissão, de $10 \mathrm{mg} \cdot \mathrm{Nm}^{-3}$.

As PCDD/F possuem duas vias de formação conhecidas. A primeira é com base em precursores e a segunda por meio da síntese de novo (AURELL; MARKLUND, 2009; VEHLOW, 2012). A primeira ocorre dentro da câmara de combustão, por meio de reações heterogêneas entre sólido e gás, tendo como precursores moléculas orgânicas cloradas e clorofenóis (AURELL; MARKLUND, 2009; VEHLOW, 2012). A segunda, principal via de formação de PCDD/F durante o processo de incineração de resíduos, é a síntese de novo (VEHLOW, 2012).

Essa síntese ocorre na região posterior da caldeira, por ser uma região onde há redução das temperaturas dos gases de combustão, que se encontram em torno de 200 a $250^{\circ} \mathrm{C}$ (VEHLOW, 2012). Os principais precursores dessa reação são fontes de carbono provenientes da combustão incompleta, como, por exemplo, carbono particulado e fuligem. São necessários atmosfera oxidante e presença de cloretos inorgânicos como fonte de cloro (VEHLOW, 2012).

Outros dois compostos encontrados nos gases de combustão, enxofre e halogênios, interferem na formação de PCDD/F. Os halogênios não aumentam a formação de PCDD/F desde que haja combustão completa. Já o enxofre, em níveis altos e constantes, inibe a formação de dioxinas. Como sua concentração nos gases é muito variável, é sugerida a recirculação do $\mathrm{SO}_{2}$ retirado da etapa de lavagem no suprimento de ar secundário.

Por serem compostos potencialmente tóxicos, $\mathrm{PCDD} / \mathrm{F}$ possuem maior restrição no tocante ao limite de emissão pela Diretiva 2010/75/UE, de 0,1 ngTE.Nm ${ }^{-3}$. Apesar de a legislação regulamentar as PCDD/F como parâmetros a serem medidos periodicamente, o estudo do seu perfil de emissão é importante mediante a preocupação com a saúde pública.

Nos incineradores analisados, conforme apresentado na Figura 4, o maior valor médio anual de emissões de PCDD/F $\left(0,02\right.$ ngTE. $\left.^{-3}\right)$ foi observado em 2016, e o menor valor médio anual $\left(0,007\right.$ ngTE. $\left.\mathrm{Nm}^{-3}\right)$ em 2014. Esses valores correspondem, respectivamente, a duas e oito vezes menos do que o limite permitido pela Diretiva da Comunidade Europeia. Em 2012 e 2016, ocorreram

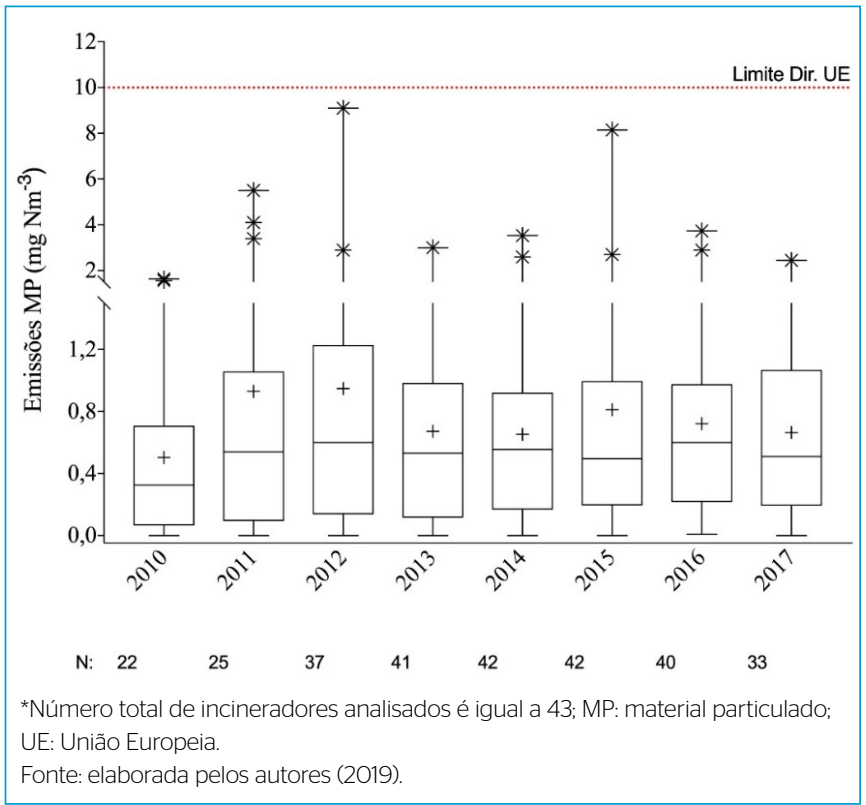

Figura 3 - Distribuição dos valores de emissão do material particulado*. medidas de emissão acima do limite permitido em dois diferentes incineradores. Os valores médios de PCDD/F, nos anos avaliados, estão em torno de $80 \%$ abaixo do referido limite legal de emissão $\left(0,1\right.$ ngTE.Nm $\left.{ }^{-3}\right)$.

A observação das emissões nos oito anos avaliados mostra que os valores de concentração de poluentes se mantêm, de forma contínua, muito abaixo dos limites estabelecidos pela legislação. Identificam-se apenas pequenas variações nas emissões ao longo do tempo.

Uma análise mais ampla, reunindo os poluentes de monitoramento contínuo, $\mathrm{PCDD} / \mathrm{F}$ e os demais poluentes de monitoramento periódico (metais, cádmio, tálio e mercúrio) definidos na Diretiva 2010/75/EU foi realizada para o período de 2015 a 2017.

Na Tabela 1, são apresentadas as médias anuais de concentração para os 11 poluentes de controle, segundo a Diretiva europeia, no período de 2015 a 2017, além dos valores máximos permitidos para emissões (UE, 2010). Para os três anos estudados, o grupo de incineradores analisado apresentou valores médios de emissões, para todos os parâmetros, abaixo dos limites permitidos. É possível ainda notar um comportamento constante nos valores das emissões ao longo dos anos.

Os poluentes de monitoramento periódico são amplamente estudados na literatura. Estão presentes em diversos resíduos sólidos encaminhados ao incinerador. Os resíduos eletrônicos são responsáveis principalmente pela emissão de cobre e chumbo (GULLETT et al., 2007), papel e plástico pela emissão de chumbo e cádmio e as baterias pela emissão de cádmio (HASSELRIIS; LICATA, 1996). Já o cromo é emitido pela queima de papel de jornal, filme plástico e resíduos têxteis (HASSELRIIS; LICATA, 1996).

Durante o processo de combustão, esses metais podem ser total ou parcialmente evaporados, estando presentes tanto nos gases quanto nas cinzas volantes (EUROPEAN COMISSION, 2006). Abanades et al. (2002) e Zhang et al. (2008) classificam os metais em três grupos principais, de acordo com sua termodinâmica e outras similaridades: cádmio e mercúrio, que possuem alta vaporização durante a incineração, principalmente como cloretos; arsênio é parcialmente volatilizado, mas tem maior tendência em estar presente nas escórias; cromo, cobre, cobalto e níquel são encontrados sobretudo nas escórias.

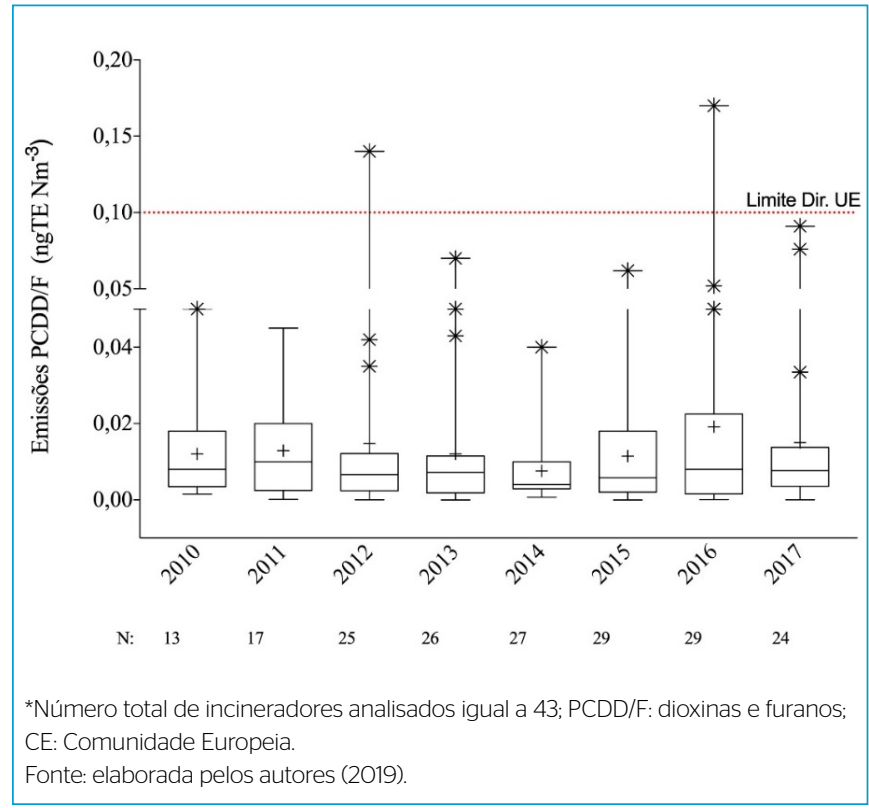

Figura 4 - Distribuição dos valores de emissão de dioxinas e furanos*. 
A separação dos metais por maior concentração nas escórias, cinzas volantes ou nos gases de combustão está relacionada à especiação e partição. A especiação refere-se às formas químicas dos elementos, enquanto a partição se refere à distribuição desses metais entre os vários fluxos de matérias que deixam o incinerador (ABANADES et al., 2002).

A especiação é influenciada pelas condições de operação da combustão, e tanto a especiação quanto a partição são influenciadas pela presença de compostos reativos, como cloro e enxofre (ABANADES et al., 2002; ZHANG et al., 2008). O cloro, por exemplo, é inicialmente oxidado a cloreto de hidrogênio $(\mathrm{HCl})$ que, por sua vez, auxilia a vaporização dos metais por meio da formação de cloretos metálicos gasosos com alta pressão de vapor (JIAO et al., 2013). Já os óxidos de enxofre, $\mathrm{SO}_{2}$ e $\mathrm{SO}_{3}$, têm potencial de alterar a partição de vapor dos metais, por meio da transformação de cloretos em sulfatos (JIAO et al., 2013).

Font et al. (2015) estudaram as emissões de incineradores na Inglaterra e, entre os metais, os que se apresentaram em maiores quantidades foram cromo $(\mathrm{Cr})$, cádmio $(\mathrm{Cd})$, níquel $(\mathrm{Ni})$, chumbo $(\mathrm{Pb})$ e cobre $(\mathrm{Cu})$, quando comparados ao controle, ou seja, a emissões amostradas em uma área rural. A avaliação das emissões foi realizada no período entre 2003 e 2010. Para cádmio, o valor da mediana encontrado foi de $0,0013 \mathrm{mg} \cdot \mathrm{m}^{-3} \mathrm{e}$ seu máximo de emissão foi de $0,026 \mathrm{mg} \cdot \mathrm{m}^{-3}$.

No presente trabalho, foram consideradas as emissões de cádmio em conjunto com tálio, a mesma forma adotada pela Diretiva europeia e na qual os incineradores divulgam em seus relatórios. Ainda assim, os valores aqui observados foram inferiores ao descrito por Font et al. (2015).

Outro metal de grande importância ambiental e para a saúde pública é o mercúrio, pelo seu potencial de bioacumulação e biomagnificação, principalmente na forma de metilmercúrio (ZHU; ZHANG; ŽAGAR, 2018). Importantes fontes de emissão atmosférica de mercúrio, de origem antropogênica, são as plantas de geração de energia com base em combustíveis fósseis (PIRRONE et al., 2009).

Em meados dos anos 90, antes de a Diretiva 2010/75 da União Europeia, relativa à incineração, ser promulgada, incineradores da Itália apresentavam medidas de mercúrio de 0,03-0,19 mg.Nm³ (LIBERTI et al., 1998). Atualmente, com o limite legal mais restritivo, as emissões de mercúrio, nas amostras de incineradores analisadas neste trabalho, resultam em valores médios anuais muito abaixo de $0,05 \mathrm{mg} \cdot \mathrm{Nm}^{-3}$.
Damgaard et al. (2010) mostraram a variação das emissões dos poluentes de acordo com o tipo de tratamento dos gases necessário ao longo dos anos. Como exemplo, nos anos 1970 e 1980, os valores de emissões de $\mathrm{HCl}$ eram em torno de 3.800 g.ton ${ }^{-1}$ de resíduo. Nesse período, os equipamentos de tratamento de gases tinham somente a função de remover as partículas sólidas. Atualmente, com a utilização de equipamentos de alta eficiência de remoção para material particulado, gases e outros poluentes, as emissões de $\mathrm{HCl}$ encontram-se em torno de 3 g.ton ${ }^{-1}$ de resíduo (DAMGAARD et al., 2010).

É possível notar que, ao longo do tempo, de forma a atender a limites legais cada vez mais restritivos, as unidades de incineração vêm investindo em rigoroso controle de suas condições operacionais e no aprimoramento das tecnologias de tratamento de gases, fatores que exercem influência direta nas suas emissões.

\section{Questões acerca da implementação de incineradores no Brasil}

O cenário atual da gestão de resíduos sólidos no Brasil vem passando por reformulações e modificações, em grande parte, em consequência da implementação da Política Nacional de Resíduos Sólidos (PNRS) instituída pela Lei no 12.305, de 2 de agosto de 2010 (BRASIL, 2010b). Embora seja perceptível o avanço na gestão de resíduos sólidos no país, particularmente nas regiões metropolitanas, na maioria dos municípios brasileiros ela continua bastante deficiente.

Segundo dados do Diagnóstico do Manejo de Resíduos Sólidos Urbanos 2017 do Sistema Nacional de Informações sobre Saneamento (SNIS), são destinados corretamente para aterros sanitários $64,2 \%$ da massa total de resíduos. A destinação irregular em aterros controlados ou lixões fica com 17,9\% e os $18 \%$ restantes não apresentam informações (SNIS, 2017).

Os tratamentos de RSU, como forma de redução da quantidade de resíduos enviados para aterros, além de recuperação de materiais e energia, começam a ser avaliados em alguns municípios do país, sobretudo naqueles de médio e grande porte. Esses tratamentos são demandados sobretudo em locais onde é necessário percorrer longas distâncias para dispor adequadamente os RSU em aterros sanitários e naqueles que querem se adequar à PNRS, destinando apenas rejeitos aos aterros.

A incineração de RSU é um tratamento que começa a ser considerado no cenário de gestão de resíduos no Brasil. Por meio de parcerias público-privadas, unidades de incineração já se encontram em processo de licenciamento ambiental em alguns estados do país (MANNARINO; FERREIRA; GANDOLLA, 2016).

Tabela 1 - Emissões atmosféricas dos poluentes de monitoramento contínuo e periódico no período de 2015 a 2017 , de 76 incineradores da União Europeia.

\begin{tabular}{|c|c|c|c|c|}
\hline Poluente/ Média Anual (mg× $\left.\mathrm{Nm}^{-3}\right)$ & 2015 & 2016 & 2017 & Limite UE $\left(\mathrm{mg} \times \mathrm{Nm}^{-3}\right)$ \\
\hline Óxidos de nitrogênio $\left(\mathrm{NO}_{x}\right)$ & 96,16 & 96,56 & 98,03 & 200 \\
\hline Material particulado (MP) & 0,99 & 0,91 & 0,67 & 10 \\
\hline Monóxido de carbono (CO) & 8,98 & 9,6 & 11,2 & 50 \\
\hline Dióxido de enxofre $\left(\mathrm{SO}_{2}\right)$ & 7,83 & 8,15 & 8,29 & 50 \\
\hline Carbono orgânico total (COT) & 0,87 & 0,87 & 0,88 & 10 \\
\hline Dioxinas e furanos (PCDD/F) (ngTExNm³) & 0,032 & 0,014 & 0,014 & 0,1 (ngTexNm³) \\
\hline Ácido clorídrico (HCl) & 2,49 & 2,43 & 2,43 & 10 \\
\hline Ácido fluorídrico (HF) & 0,12 & 0,1 & 0,14 & 1 \\
\hline Mercúrio (Hg) & 0,0026 & 0,0213 & 0,0194 & 0,05 \\
\hline Cádmio (Cd) + tálio (TI) & 0,0039 & 0,0018 & 0,00337 & 0,05 \\
\hline Metais (Sb + As + Pb + Cr + Co + Cu+ Mn + Ni + V) & 0,206 & 0,039 & 0,044 & 0,5 \\
\hline
\end{tabular}

EU: União Europeia.

Fonte: elaborada pelos autores (2019). 
Como aos técnicos de órgãos de controle ambiental já foi dada a responsabilidade de avaliar projetos de incineradores no país, fazem-se prementes discussões sobre a legislação vigente de controle de emissões para essa tecnologia. Em nível nacional, o Conselho Nacional do Meio Ambiente (CONAMA) conta com a Resolução no 316/2002 (BRASIL, 2002), que dispõe sobre procedimentos e critérios para o funcionamento de sistemas de tratamento térmico de resíduos.

A referida Resolução CONAMA data de mais de 15 anos atrás e estabelece valores limites de emissões atmosféricas bastante permissivos, quando comparados aos da legislação europeia. Além disso, explora pouco as condições de operação do processo de incineração e não faz referência à possibilidade de recuperação energética. Ainda, não há qualquer menção à divulgação de informações de operação e monitoramento por parte dos gestores das unidades de incineração.

A recente Portaria Interministerial no 274, de 30 de abril de 2019 (BRASIL, 2019), que disciplina a recuperação energética dos RSU, referida no $\$ 1^{\circ} \mathrm{do}$ art. $9^{\circ} \mathrm{da}$ Lei ${ }^{\circ}$ 12.305, de 2010 (BRASIL, 2010b), e no art. 37 do Decreto $n^{\circ} 7.404$, de 2010 (BRASIL, 2010a), define os tipos de resíduos que podem ser enviados a unidades de recuperação de energia e a temperatura mínima de sua operação. Essa Portaria não versa sobre valores de emissões atmosférica, ficando mantidos os padrões de emissão definidos pela Resolução CONAMA nº 316/2002 (BRASIL, 2002).

Em nível estadual, o estado do Paraná conta com a Resolução nº 043/2008 da Secretaria do Meio Ambiente e Recursos Hídricos (SEMA/PR), que dispõe sobre o licenciamento ambiental, e estabelece condições e critérios para empreendimentos de incineração de resíduos sólidos (PARANÁ, 2008). A Resolução $\mathrm{n}^{\circ}$ 016/2014, da mesma Secretaria, estabelece os padrões de emissão atmosférica especificamente para incineradores (PARANÁ, 2014). No entanto, seus valores de referência para emissões atmosféricas também são permissivos e semelhantes aos da Resolução CONAMA n 316/2002 (BRASIL, 2002).

O estado de São Paulo também possui legislação específica sobre o tratamento térmico de resíduos. Sua Secretaria de Estado do Meio Ambiente
(SMA/SP) promulgou a Resolução SMA nº 79/2009, que estabelece diretrizes e condições para a operação e licenciamento de resíduos sólidos em usinas de recuperação de energia (SÃO PAULO, 2009). Essa resolução define valores limites para emissões atmosféricas mais restritos do que os das Resoluções CONAMA n 316/2002 (BRASIL, 2002) e SEMA/PR 043/2008 (PARANÁ, 2008), estando próximos ou idênticos aos estabelecidos pela Diretiva 2010/75 da União Europeia. A Tabela 2 mostra a comparação entre as resoluções brasileiras citadas e a Diretiva europeia.

Uma fragilidade percebida na gestão de resíduos brasileira é o acesso aos dados e informações relativas a geração, tratamento, destino final, entre outros. No que diz respeito à incineração, a divulgação de dados de monitoramento de emissões atmosféricas, efluentes, cinzas e escórias é de grande importância para que a sociedade tenha conhecimento dos reais impactos da atividade e da possibilidade de fiscalização. A incineração pode ser considerada uma atividade segura do ponto de vista ambiental, se operada mediante padrões rigorosos de controle, mas também pode ser uma fonte de impacto significativa, caso seus subprodutos não atendam a limites seguros de concentrações de poluentes.

Além do monitoramento ambiental, o biomonitoramento (com o uso de organismos vivos) prévio e contínuo no entorno de incineradores é fundamental para garantir o controle dos impactos ao ambiente e à saúde pública. Instituições de pesquisa e institutos idôneos devem ter papel importante na geração de dados e no acompanhamento de impactos da atividade de incineração.

Cabe ainda ressaltar que o controle dos gases e outros subprodutos gerados em incineradores de RSU está diretamente ligado à estabilidade do processo de combustão e dos tratamentos adotados. Essa estabilidade é obtida, entre outros fatores, por meio da continuidade operacional. Incineradores de RSU devem funcionar 24 horas por dia, com paradas apenas em manutenções preventivas anuais programadas ou em casos de falhas eventuais. A continuidade operacional de uma unidade de incineração precisa ser mantida, independentemente de mudanças na sua gestão.

Tabela 2 - Comparação entre limites de emissão de poluentes atmosféricos das legislações europeia e brasileiras para incineradores de RSU.

\begin{tabular}{|c|c|c|c|c|}
\hline Parâmetro $\left(\mathrm{mg} \mathrm{Nm}^{-3}\right)^{*}$ & Diretiva 2010/75 (UE) & $\begin{array}{c}\text { Resolução CONAMA } \\
n^{\circ} 316 / 2002\end{array}$ & $\begin{array}{c}\text { Resolução SEMA } \\
\text { 043/2008 (PR) }\end{array}$ & $\begin{array}{c}\text { Resolução SMA } \\
\text { 79/2009 (SP) }\end{array}$ \\
\hline Óxidos de nitrogênio $\left(\mathrm{NO}_{x}\right)$ & 200 & 560 & 560 & 200 \\
\hline Material particulado (MP) & 10 & 70 & 70 & 10 \\
\hline Monóxido de carbono (CO) & 50 & 100 & 125 & 50 \\
\hline Dióxido de enxofre $\left(\mathrm{SO}_{2}\right)$ & 50 & 280 & 280 & 50 \\
\hline Carbono orgânico total (COT) & 10 & - & - & 10 \\
\hline Ácido clorídrico $(\mathrm{HCl})$ & 10 & 80 & 80 & 10 \\
\hline Ácido fluorídrico (HF) & 1 & 5 & 5 & 1 \\
\hline Dioxinas e furanos (PCDD/F) (ngTE.Nm³) & 0,1 & 0,5 & 0,5 & 0,1 \\
\hline mercúrio $(\mathrm{Hg})$ & 0,05 & \multirow{3}{*}{0,28} & \multirow{3}{*}{0,28} & 0,05 \\
\hline cádmio (Cd) & \multirow{2}{*}{0,05} & & & \multirow{2}{*}{0,05} \\
\hline tálio (TI) & & & & \\
\hline $\mathrm{Sb}+\mathrm{As}+\mathrm{Pb}+\mathrm{Cr}+\mathrm{Co}+\mathrm{Cu}+\mathrm{Mn}+\mathrm{Ni}+\mathrm{V}$ & 0,5 & - & - & 0,5 \\
\hline $\mathrm{Sb}+\mathrm{Pb}+\mathrm{Cr}+\mathrm{Cn}+\mathrm{Cu}+\mathrm{Sn}+\mathrm{F}+\mathrm{Mn}+\mathrm{PT}+\mathrm{PD}+\mathrm{RH}+\mathrm{V}$ & - & 7 & 7 & - \\
\hline $\mathrm{As}+\mathrm{Co}+\mathrm{Ni}+\mathrm{Se}+\mathrm{Te}$ & - & 1,4 & 1,4 & - \\
\hline
\end{tabular}

*Exceto dioxinas e furanos; RSU: resíduos sólidos urbanos; EU: União Europeia; CONAMA: Conselho Nacional do Meio Ambiente; SEMA: Secretaria do Meio Ambiente e Recursos Hídricos; SMA: Secretaria de Estado do Meio Ambiente.

Fonte: elaborada pelos autores (2019) com base em UE (2010), Brasil (2002), Paraná (2008), São Paulo (2009). 


\section{CONCLUSÕES}

O Brasil possui regiões metropolitanas intensamente urbanizadas e densamente povoadas, que colocam em evidência as fragilidades da gestão de RSU no país. Processos de tratamento de resíduos, como a incineração, começam a se ser considerados como alternativas, visto que, por meio da redução de massa e volume dos resíduos, eles otimizam a vida útil de aterros e possibilitam a recuperação de energia.

O trabalho buscou analisar dados de monitoramento ambiental de incineradores em operação na Europa, onde essa é uma tecnologia consolidada na gestão de resíduos. Na UE, a incineração é regulada pela Diretiva 2010/75, que estipula formas de operação e controle dos processos, bem como limites de emissões atmosféricas. A Diretiva da UE estabelece a obrigatoriedade da divulgação dessas informações para a sociedade, pelos operadores de plantas de incineração.

Verificou-se que a comunicação dos dados e informações técnicas pode ser um bom instrumento de controle social sobre a operação dos incineradores. Todos os dados utilizados neste trabalho foram coletados nos websites dos respectivos incineradores. Tanto informações técnicas sobre a planta - como capacidade de recepção de resíduos, tipo de forno e tipos de resíduos recebidos quanto dados de monitoramento de emissões atmosféricas estão disponíveis à sociedade, principalmente na forma de relatórios anuais de monitoramento.

Os dados de monitoramento mostram que as concentrações de poluentes nos gases emitidos por incineradores na Europa tendem a estar muito abaixo dos limites estabelecidos por lei e a ter comportamento constante ao longo dos anos. Com base nos dados publicados pelas plantas analisadas, pode-se inferir que a incineração é uma tecnologia de tratamento térmico de RSU com reduzida contribuição para a poluição atmosférica, desde que bem operada e monitorada. Legislações bem estruturadas são decisivas para balizar a operação da tecnologia.

Dessa forma, a possível implementação de incineradores no Brasil precisa ser precedida da definição legal de parâmetros de controle e monitoramento ambiental rigorosos, bem como da exigência de que dados e informações acerca de emissões sejam públicos, com acesso garantido à população. Faz-se necessária a reformulação da legislação nacional vigente, antiga e permissiva, tomando por referência exigências já estabelecidas por países desenvolvidos. É imprescindível que se assegurem padrões de controle bem definidos e a continuidade operacional das unidades para que essa tecnologia possa ser utilizada com segurança nos sistemas de gestão de RSU no país.

\section{CONTRIBUIÇõES DOS AUTORES}

Cardozo, B. C.: Conceituação, Investigação, Análise Formal, Escrita Primeira Redação. Mannarino, C. F.: Conceituação, Supervisão, Validação, Escrita - Revisão e Edição. Ferreira, J. A.: Conceituação, Supervisão, Validação, Escrita - Revisão e Edição.

\section{REFERÊNCIAS}

ABANADES,S.FLAMANT,G.;GAGNEPAIN,B.;GAUTHIER,D.Fateof HeavyMetals during Municipal Solid Waste Incineration. Waste Management \& Research, v. 20, n. 1, p. 55-68, 2002. https://doi.org/10.1177/0734242X0202000107

AURELL, J.; MARKLUND, S. Effects of varying combustion conditions on PCDD/F emissions and formation during MSW incineration. Chemosphere, v. 75, n. 5, p. 667-673, 2009. https://doi.org/10.1016/j.chemosphere.2008.12.038

BRASIL. Conselho Nacional do Meio Ambiente (CONAMA). Resolução no 316, de 29 de outubro de 2002. Dispõe sobre procedimentos e critérios para o funcionamento de sistemas de tratamento térmico de resíduos. Correlações: Artigo 18 alterado pela Resolução Conama no 386/06. Diário Oficial da União, Brasília, n. 224, Seção 1, p. 92-95, 20 nov. 2002.

BRASIL. Decreto no 7.404, de 23 de dezembro de 2010. Regulamenta a Lei no 12.305, de 2 de agosto de 2010, que institui a Política Nacional de Resíduos Sólidos, cria o Comitê Interministerial da Política Nacional de Resíduos Sólidos e o Comitê Orientador para a Implantação dos Sistemas de Logística Reversa, e dá outras providências. Diário Oficial da União, Brasilia, Edição Extra, p. 1, 23 dez. 2010a.

BRASIL. Lei no 12.305, de 2 de agosto 2010. Institui a Política Nacional de Resíduos Sólidos. Altera a Lei no 9.605, de 12 de fevereiro de 1998, e dá outras providências. Diário oficial [da] República Federetiva do Brasil, Brasília, Seção 1, p. 3, 3 ago. 2010b.

BRASIL. Portaria Interministerial no 274, de 30 de abril de 2019. Disciplina a recuperação energética dos resíduos sólidos urbanos referida no $\S 10$ do art. $9^{\circ}$ da Lei no 12.305, de 2010 e no art. 37 do Decreto no 7.404, de 2010. Diário Oficial da União, Brasília, Edição 83, Seção 1, p. 57, 2 maio 2019.
BUONANNO, G.; FICCO, G.; STABILE, L. Size distribution and number concentration of particles at the stack of a municipal waste incinerator. Waste Management, v. 29, n. 2, p. 749-755, 2009. https://doi.org/10.1016/j. wasman.2008.06.029

BUONANNO, G.; STABILE, L.; AVINO, P.; BELLUSO, E. Chemical, dimensional and morphological ultrafine particle characterization from a waste-toenergy plant. Waste Management, v. 31, n. 11, p. 2253-2262, 2011. https://doi org/10.1016/j.wasman.2011.06.017

CARBONE, F; BARONE, A.; PAGLIARA, R.; BERETTA, F.; D'ANNA, A.; D'ALESSIO, A. Ultrafine Particles Formed by Heating Droplets of Simulated Ash Containing Metals. Environmental Engineering Science, v. 25, n. 10, p. 1379-1388, 2008. https://doi.org/10.1089/ees.2007.0190

CASS, G.R.; HUGHES, L.A.; BHAVE, P.; KLEEMAN, M.J.; ALLEN, J.O.; SALMON L.G. The chemical composition of atmospheric ultrafine particles. Philosophical Transactions of the Royal Society A, v. 358, n. 1775, p. 2581 2592, 2000. https://doi.org/10.1098/rsta.2000.0670

CONFEDERATION OF EUROPEAN WASTE-TO-ENERGY PLANTS (CEWEP). CEWEP Energy Report III. Düsseldorf: CEWEP, 2017. Disponível em: https:/l www.cewep.eu/. Acesso em: 6 fev. 2021.

DAMGAARD, A.; RIBER, C.; FRUERGAARD, T.; HULGAARD, T.; CHRISTENSEN, T.H. Life-cycle-assessment of the historical development of air pollution control and energy recovery in waste incineration. Waste Management, v. 30, n. 7. p. 1244-1250, 2010. https://doi.org/10.1016/j. wasman.2010.03.025 
EUROPEAN COMMISSION. Best Available Techniques for the Management of Waste from Extractive Industries in accordance with Directive 2006/21/EC. European Commission, 2006. Disponivel em: https:/ec.europa.eu/jrc/en/publication/eurscientific-and-technical-research-reports/best-available-techniques-bat-referencedocument-management-waste-extractive-industries. Acesso em: 27 jan. 2021.

FONT, A.; HOOGH, K.; LEAL-SANCHEZ, M.; ASHWORTH, D.C.; BROWN, R.J.C.; HANSELL, A.L.; FULLER, G.W. Using metal ratios to detect emissions from municipal waste incinerators in ambient air pollution data. Atmospheric Environment,v.113,p.177-186,2015.https://doi.org/10.1016/j.atmosenv.2015.05.002

GULLETT, B.K.; LINAK, W.P.; TOUATI, A.; WASSON, S.J.; GATICA, S.; KING, C.J. Characterization of air emissions and residual ash from open burning of electronic wastes during simulated rudimentary recycling operations. Journal of Material Cycles and Waste Management, v. 9, n. 1, p. 69-79, 2007. https://doi.org/10.1007/s10163-006-0161-x

HASSELRIIS, F; LICATA, A. Analysis of heavy metal emission data from municipal waste combustion. Journal of Hazardous Materials, v. 47, n. 1-3, p. 77-102, 1996. https://doi.org/10.1016/0304-3894(95)00107-7

JIAO, F.; ZHANG, L.; YAMADA, N.; SATO, A.; NINOMIYA, Y. Effect of HCl, SO2 and $\mathrm{H} 2 \mathrm{O}$ on the condensation of heavy metal vapors in flue gas cooling section. Fuel Processing Technology, v. 105, p. 181-187, 2013. https://doi. org/10.1016/j.fuproc.2011.06.024

KUMAR, P.; PIRJOLA, L.; KETZEL, M.; HARRISON, R.M. Nanoparticle emissions from 11 non-vehicle exhaust sources: a review. Atmospheric Environment, v. 67. p. 252-277, 2013. https://doi.org/10.1016/j.atmosenv.2012.11.011

LIBERTI, L:; NOTARNICOLA, M; AMICARELLI, V:; CAMPANARO, V: ROETHEL, F; SWANSON, L. Mercury removal with powdered activated carbon from flue gases at the Coriano municipal solid waste incineration plant. Waste Management and Research, v. 16, n. 2, p. 183-189, 1998. https://doi.org/10.1177\%2FO734242X9801600210

MANNARINO, C.F.; FERREIRA, J.A.; GANDOLLA, M. Contribuições para a evolução do gerenciamento de resíduos sólidos urbanos no Brasil com base na experiência Européia. Engenharia Sanitária e Ambiental, v. 21, n. 2 , p. 379-385, 2016. https://doi.org/10.1590/s1413-41522016146475

NIXON, J.D.; WRIGHT, D.G.; DEY, P.K.; GHOSH, S.K.; DAVIES, P.A. A comparative assessment of waste incinerators in the UK. Waste Management, v. 33, n. 11, p. 2234-2244, 2013. https://doi.org/10.1016/j.wasman.2013.08.001

NZIHOU, A.; THEMELIS, N.J.; KEMIHA, M.; BENHAMOU, Y. Dioxinemissions from municipal solid waste incinerators (MSWIs) in France. Waste Management, v. 32, n. 12, p. 2273-2277, 2012. https://doi.org/10.1016/j.wasman.2012.06.016

PARANÁ (Estado). Resolução SEMA no 16 de 26 de março de 2014. Define critérios para o controle de qualidade do ar como um dos instrumentos básicos da gestão ambiental para proteção da saúde e bem estar da população e melhoria da qualidade de vida. Diário Oficial do Estado do Paraná, Paraná, p. 9187, 15 abr. 2014.

PARANÁ (Estado). Resolução SEMA no 43 de 16 de julho de 2008. Dispõe sobre o licenciamento ambiental, estabelece condições e critérios para Empreendimentos de incineração de resíduos sólidos e dá outras providências. Diário Oficial do Estado do Paraná, Paraná, 25 jul. 2008.

PIRRONE, N.; CINNIRELLA, S.; FENG, X.; FINKELMAN, R.B.; FRIEDLI, H.R.; LEANER, J.; MASON, R.; MUKHERJEE, A.B.; STRACHER, G.; STREETS, D.G.; TELMER, K. Global mercury emissions to the atmosphere from natural and anthropogenic sources. In: MASON, R.; PIRRONE, N. (org.). Mercury Fate and Transport in the Global Atmosphere: Emissions, Measurements and Models. Boston: Springer, 2009. p. 3-49. https://doi.org/10.1007/978-0-387-93958-2_1
QUICKER, P.; HEE, J. Clean Incineration: Fact or Fiction? Waste Management World, p. 10-14, 2013

SÃO PAULO (Estado). Resolução SMA no 79 de 4 de novembro de 2009. Estabelece diretrizes e condições para a operação e licenciamento de resíduos sólidos em usinas de recuperação energética - URE. Diário Oficial do Estado de São Paulo, São Paulo, Executivo I, p. 44, 5 nov. 2009.

SCHNEIDER, D.R.; RAGOSSNIG, A.M. Biofuels from waste. Waste Management and Research, v. 31, n. 4, p. 339-340, 2013. https://doi. org/10.1177\%2F0734242X13482228

SISTEMA NACIONAL DE INFORMAÇÕES SOBRE SANEAMENTO (SNIS). Diagnóstico do Manejo de Resíduos Sólidos Urbanos - 2017. Brasilia: Ministério das Cidades, 2017. Disponível em: http://www.snis.gov.br. Acesso em: 6 fev. 2021.

STROBEL, R.; WALDNER, M.H.; GABLINGER, H. Highly efficient combustion with low excess air in a modern energy-from-waste (EfW) plant. Waste Management, v. 73, p. 301-306, 2018. https://doi.org/10.1016/j. wasman.2017.06.049

SVOBODA, K.; BAXTER, D.; MARTINEC, J. Nitrous oxide emissions from waste incineration. Chemical Papers, v. 60, n. 1, p. 78-90, 2006. https://doi. org/10.2478/s11696-006-0016-x

UNIÃO EUROPEIA (UE). Diretiva 2010/75/EC - Relativa às emissões industriais. Conselho da União Europeia, 2010.

USHIMA, A.H.; SANTOS, M.M. Processamento do Lixo - Parte 9 - Tratamento térmico. In: COMPROMISSO EMPRESARIAL PARA A RECICLAGEM (CEMPRE). Lixo Municipal: Manual de Gerenciamento Integrado. 4. ed. São Paulo: CEMPRE, 2018. p. 193-218.

VAN DIJK, C.; VAN DOORN, W:; VAN ALFEN, B. Long term plant biomonitoring in the vicinity of waste incinerators in The Netherlands. Chemosphere, $\mathrm{v}$. 122, p. 45-51, 10 mar. 2015. https://doi.org/10.1016/j.chemosphere.2014.11.002

VEHLOW, J. Reduction of dioxin emissions from thermal waste treatment plants : a brief survey. Reviews in Environmental Science and Biotechnology. n. 11, p. 393-405, 2012. https://doi.org/10.1007/s11157-012-9296-5

VEHLOW, J.; DALANGER, S. Incineration: Flue Gas Cleaning and Emissions. In: CHRISTENSEN, T.H. (org.). Solid Waste Tecnology \& Management. Blackwell, 2011

WALDNER, M.H.; HALTER, R.; SIGG, A.; BROSCH, B.; GEHRMANN, H.J.; KEUNECKE, M. Energy from Waste - Clean, efficient, renewable: Transitions in combustion efficiency and $\mathrm{NO}_{\mathrm{x}}$ control. Waste Management, v. 33, n. 2, p. 317-326, 2013. https://doi.org/10.1016/j.wasman.2012.08.007

WISSING, F; WIRTZ, S.; SCHERER, V. Simulating municipal solid waste incineration with a DEM/CFD method - Influences of waste properties, grate and furnace design. Fuel, v. 206, p. 638-656, 2017. https://doi.org/10.1016/j. fuel.2017.06.037

ZHANG, Y.; CHEN, Y.; MENG, A.; LI, Q.; CHENG, H. Experimental and thermodynamic investigation on transfer of cadmium influenced by sulfur and chlorine during municipal solid waste (MSW) incineration. Journal of Hazardous Materials, v. 153, n. 1-2, p. 309-319, 2008. https://doi.org/10.1016/j. jhazmat.2007.08.054

ZHU, S.; ZHANG, Z.; ŽAGAR, D. Mercury transport and fate models in aquatic systems: A review and synthesis. Science of the Total Environment, v. 639, p. 538-549, 2018. https://doi.org/10.1016/j.scitotenv.2018.04.397 
Anexo 1 - Identificação dos incineradores analisados, por país.

\begin{tabular}{|c|c|}
\hline Países & Incineradores \\
\hline Alemanha & $\begin{array}{l}\text { KIEL Die Müllverbrennung Kiel GmbH \& Co. KG (MVK), EEW Energy from Waste Hannover GmbH, EEW Energy from Waste } \\
\text { Saarbrücken GmbH, MHKW Berlin-Ruhleben, Das Müllheizkraftwerk (MHKW) Bremen, MVA Bielefeld, TREA Breisgau, MHKW Rothensee, } \\
\text { MHKW Müllheizkraftwerk Frankfurt am Main GmbH, MHKW Schwandorf. }\end{array}$ \\
\hline Andorra & Centre de Tractment de Residus d'Andorra, SA (CTRASA). \\
\hline Áustria & $\begin{array}{l}\text { EVN - Abfallverwertung Niederösterreich Gesellschaft m.b.H., KRV Kärntner Restmüllverwertungs GmbH, Spittelau, Flötzersteig, Pfaffenau, } \\
\text { WAV Wels, Zistersdorf. }\end{array}$ \\
\hline Bélgica & Oostende (IVOO), IMOG, Gent (Ivago), Ipalle Thumaide, Brussels, Beveren Indaver DOEL, ISVAG, Pont-de-Loup, Houthalen-Helchteren, Herstal. \\
\hline Dinamarca & $\begin{array}{c}\text { Bornholms Affaldsbehandling (BOFA), I/S REFA, Næstved, Vestforbrænding, Sønderborg Kraftvarmeværk I/S, Esbjerg, Thisted, I/S Reno-Nords } \\
\text { forbrændingsanlæg, I/S KAVO, TAS I/S. }\end{array}$ \\
\hline Eslováquia & Košice, Bratislava. \\
\hline Espanha & $\begin{array}{l}\text { Melilla, Consell de Mallorca, Tarragona, La Planta de Valorització Energètica (PVE) de Sant Adrià de Besò, Planta incineradora Del Maresme, } \\
\text { La planta incineradora de Campdorà, Bilbao, Meruelo, Cantabria. }\end{array}$ \\
\hline Estônia & Iru. \\
\hline Finlândia & Korkeakoski, Helsinki Vantaan Energia, Laanila, Tammervoima, Westenergy Oy Ab. \\
\hline França & $\begin{array}{c}\text { Halluin Energy Valuation Center (Antarès), Rouen, Brest Energie, Rennes, Arc- en -Ciel, D'IVRY-PARIS XIII, Valorly, SONITHERM, For Sur Mer, } \\
\text { UVE Benesse maremne. }\end{array}$ \\
\hline Holanda & Reststoffen Energie Centrale (REC) Harlingen, EEW Energy from Waste Delfzill B.V., Afvalenergiecentrale Moerdijk, Twence, Wuert, Baviro, Dordebrecht. \\
\hline Hungria & Budapest. \\
\hline Inglaterra & $\begin{array}{l}\text { Plymouth, Newhaven, Belvedere, Birmingham, Eastcroft Energy from Waste Facility, Ferrybridge, Severnside Energy Recovery Centre (SERC), } \\
\text { Suffolk, Coventry \& Solihull Waste Disposal Company (CSWDC), Marchwood. }\end{array}$ \\
\hline Irlanda & Meath. \\
\hline Itália & Acerra, Poggibonsi, Coriano, Livorno, Granarolo Emilia, Parma, Brescia, Turin, Bolzano Bozen, Errera Trieste, Piacenza. \\
\hline Lituânia & Iru. \\
\hline Noruega & Kristiansand, Oslo, BIR Avfallsenergi AS, Trondheim - Heimdal, Ora Fredrikstad, Ål. \\
\hline Polônia & Bydgoszcz, Poznań, Konin, Krakow, Gwarków, Bialystok. \\
\hline Portugal & Iru, Maia. \\
\hline República Checa & ZEVO, SAKO Brno, a.s. (Sdružení STEO), PIzeň, Termizo, a.s. Liberec. \\
\hline Suécia & Åmotfors, Dåva kraftvärmeverk, Sävenäs avfallskraftvärmeverk, Ljungsjöverket, Korsta kraftvärmeverk, Sysav förbränningsanläggning, Värmekällan \\
\hline Suíça & $\begin{array}{c}\text { Lausanne Tridel, Giubiasco ACR, Thun AVAG, La Chaux-de-Fonds VADEC, Zuchwil Emmenspitz KEBAG, Basel IWB, Perlen Renergia, Buchs (AG) } \\
\text { GEKAL, Zürich Hagenholz ERZ, Weinfelden KVA Thurgau. }\end{array}$ \\
\hline
\end{tabular}

Fonte: adaptado de Cewep (2017). 